\title{
Labyrinthe
}

40 | 2013

Comme les abeilles

\section{La proximité entre l'homme et l'abeille : un point de vue biologique}

Janine Kievits

\section{(2) OpenEdition}

1 Journals

Édition électronique

URL : http://journals.openedition.org/labyrinthe/4303

DOI : $10.4000 /$ labyrinthe.4303

ISSN : 1950-6031

Éditeur

Hermann

Édition imprimée

Date de publication : 1 mars 2013

Pagination : 33-35

ISBN : 9782705688400

\section{Référence électronique}

Janine Kievits, «La proximité entre l'homme et l'abeille : un point de vue biologique », Labyrinthe [En ligne], 40 | 2013, mis en ligne le 01 mars 2015, consulté le 19 avril 2019. URL : http:// journals.openedition.org/labyrinthe/4303 ; DOI : 10.4000/labyrinthe.4303

Propriété intellectuelle 


\title{
La proximité entre l'homme et l'abeille : un point de vue biologique
}

\author{
Janine KIEVITS
}

Que l'on s'attache à mettre en lumière une forme d'humanité chez l'abeille ou que l'on observe dans l'espèce humaine des comportements d'essaims, la volonté de rapprocher les deux espèces est coutumière et récurrente. Ne faut-il voir dans ces déclarations de proximités, cet intérêt et cette fascination pour l'abeille qu'anthropomorphisme simpliste, rêveries champêtres de poètes dominicaux ou fantaisies puériles nourries d'images d'Épinal et de dessins animés japonais ? Le rapprochement n'est pourtant pas simple projection, et repose sur des fondements biologiques.

Partons d'un constat: les deux espèces sont ubiquistes, elles sont parvenues à s'implanter et s'étendre sous toutes les latitudes ${ }^{1}$, ce qui suppose, dans les deux cas, de grandes facultés d'adaptation. On peut en dénombrer quatre principales: résister aux variations de températures; savoir constituer des réserves de nourriture, pour survivre lors des périodes où la récolte est impossible; parvenir, grâce à des compétences cognitives, à se procurer des moyens de survies dans tous les environnements (songeons aux abeilles sahariennes ou arctiques); et protéger ses jeunes pour assurer la survie de l'espèce. Toutes qualités qui impliquent la mémoire, l'apprentissage, la capacité d'expérimenter.

Il est dès lors possible de reprendre la comparaison point par point. La température: l'être humain maintient celle de son corps autour de $37^{\circ} \mathrm{C}$, et affronte celle de l'extérieur à la fois grâce à ses vêtements et grâce à son logement; s'il n'y a pas chez l'abeille de température interne constante (elle peut varier de 7 à $50^{\circ} \mathrm{C}$ ), il y a bien une régulation au niveau de la ruche entre 34 et $36^{\circ} \mathrm{C}$, principalement au moment où les larves grandissent. Les stocks : les hommes savent en constituer, et n'hésitent pas à se faire la guerre pour se les approprier; il en va de même pour les

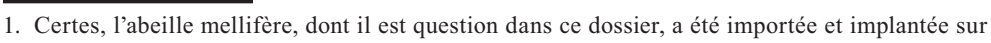
le continent américain; mais une fois dans la place, elle l'a rapidement et totalement colonisée. 


\section{Labyrinthe, $n^{\circ} 40$}

abeilles, qui, en cas de pénurie, peuvent s'organiser en colonies pillardes qui patrouillent à la recherche de sucre. Les capacités cognitives: elles sont évidentes dans notre espèce, mais l'abeille possède également des facultés d'apprentissage et de mémoire qu'elle met notamment à profit en élaborant de véritables stratégies de butinage. Les petits: chez les humains la gestation, la nourriture spécialisée et les soins dispensés grâce aux qualités affectives permettent une protection des jeunes; les jeunes abeilles, quant à elles, sont élevées dans la zone centrale de la ruche, la plus chaude (les « berceaux »), et, si elles n'ont pas le lait maternel des vertébrés, elles profitent également d'une nourriture spécifique, la gelée royale, sécrétion des glandes, complémentée avec une bouillie de pollen et d'eau fabriquée par les abeilles nourrices.

Les parentés sont donc réelles et conséquentes. Elles appellent deux remarques: d'abord, on relèvera qu'elles n'opèrent pas nécessairement d'individu (humain) à individu (abeille), mais d'individu (humain) à colonie (d'abeilles), en une similitude asymétrique qui permet d'élaborer le concept de «super-organisme »; d'autre part, il faut noter que cette proximité entre les deux espèces ne procède en aucune manière d'une évolution commune, les deux lignées évolutives, celle des insectes et celle des mammifère, ayant très tôt divergé: les ressemblances proviennent donc de difficultés communes rencontrées par les deux espèces qui ont, par évolution, élaboré des moyens comparables, voire similaires, de les surmonter et de subvenir à leurs besoins. Dire que l'abeille est « moins évoluée » que nous n'a donc aucun sens : elle est, comme nous, un « sommet évolutif », chacun sur sa ligne respective. Dès lors on comprend mieux le sentiment de familiarité éprouvé à son endroit, le pressentiment d'une forme d'égalité qui nourrit images, fictions et mythes. Cela met aussi au jour un aspect déterminant des rapports entre l'apiculteur et l'abeille: le premier n'est pas maître des secondes, l'apiculture n'est pas un élevage, encore moins une domestication, mais bien une négociation constante avec un peuple autonome. 
Instinct de la construction des cellules chez l'abeille. - Je n'ai pas l'intention d'entrer ici dans des détails très circonstanciés, je me contenterai de résumer les conclusions auxquelles j'ai été conduit sur ce sujet. Qui peut examiner cette délicate construction du rayon de cire, si parfaitement adapté à son but, sans éprouver un sentiment d'admiration enthousiaste? Les mathématiciens nous apprennent que les abeilles ont pratiquement résolu un problème des plus abstraits, celui de donner à leurs cellules, en se servant d'une quantité minima de leur précieux élément de construction, la cire, précisément la forme capable de contenir le plus grand volume de miel. Un habile ouvrier, pourvu d'outils spéciaux, aurait beaucoup de peine à construire des cellules en cire identiques à celles qu'exécutent une foule d'abeilles travaillant dans une ruche obscure. Qu'on leur accorde tous les instincts qu'on voudra, il semble incompréhensible que les abeilles puissent tracer les angles et les plans nécessaires et se rendre compte de l'exactitude de leur travail. La difficulté n'est cependant pas aussi énorme qu'elle peut le paraître au premier abord, et l'on peut, je crois, démontrer que ce magnifique ouvrage est le simple résultat d'un petit nombre d'instincts très simples.

$[\ldots]$

Ainsi, à mon avis, le plus étonnant de tous les instincts connus, celui de l'abeille, peut s'expliquer par l'action de la sélection naturelle. La sélection naturelle a mis à profit les modifications légères, successives et nombreuses qu'ont subies des instincts d'un ordre plus simple; elle a ensuite amené graduellement l'abeille à décrire plus parfaitement et plus régulièrement des sphères placées sur deux rangs à égales distances, et à creuser et à élever des parois planes sur les lignes d'intersection. Il va sans dire que les abeilles ne savent pas plus qu'elles décrivent leurs sphères à une distance déterminée les unes des autres, qu'elles ne savent ce que c'est que les divers côtés d'un prisme hexagonal ou les rhombes de sa base. La cause déterminante de l'action de la sélection naturelle a été la construction de cellules solides, ayant la forme et la capacité voulues pour contenir les larves, réalisée avec le minimum de dépense de cire et de travail. L'essaim particulier qui a construit les cellules les plus parfaites avec le moindre travail et la moindre dépense de miel transformé en cire a le mieux réussi, et a transmis ses instincts économiques nouvellement acquis à des essaims successifs qui, à leur tour aussi, ont eu plus de chances en leur faveur dans la lutte pour l'existence.

Charles Darwin, L'Origine des espèces, chap. VIII, trad. E. Barbier, Schleicher Frères éditeurs, 1906. 